\title{
Measurements of wave damping by a grease ice slick in Svalbard using off-the-shelf sensors and open-source electronics
}

\author{
JEAN RABAULT, GRAIG SUTHERLAND, OLAV GUNDERSEN, ATLE JENSEN
}

\author{
Department of Mathematics, University of Oslo, Oslo, Norway \\ Correspondence: Jean Rabault <jeanra@math.uio.no>
}

\begin{abstract}
Versatile instruments assembled from off-the-shelf sensors and open-source electronics are used to record wave propagation and damping measured by Inertial Motion Units (IMUs) in a grease ice slick near the shore in Adventfjorden, Svalbard. Viscous attenuation of waves due to the grease ice slick is clearly visible by comparing the IMU data recorded by the different instruments. The frequency dependent spatial damping of the waves is computed by comparing the power spectral density obtained from the different IMUs. We model wave attenuation using the one-layer model of Weber from 1987. The best-fit value for the effective viscosity is $v=\left(0.95 \pm 0.05 \times 10^{-2}\right) \mathrm{m}^{2} \mathrm{~s}^{-1}$, and the coefficient of determination is $R^{2}=0.89$. The mean absolute error and RMSE of the damping coefficient are 0.037 and $0.044 \mathrm{~m}^{-1}$, respectively. These results provide continued support for improving instrument design for recording wave propagation in ice-covered regions, which is necessary to this area of research as many authors have underlined the need for more field data.
\end{abstract}

KEYWORDS: frazil ice, ice/ocean interactions, sea ice

\section{INTRODUCTION}

Understanding the interaction between surface waves and sea ice is an area of ongoing research. Specific applications of eventual successful quantitative results include the formulation of ocean models for climate, weather and sea state prediction (Christensen and Broström, 2008), the estimation of ice thickness (Wadhams and Doble, 2009) and the analysis of pollution dispersion in the Arctic environment (Pfirman and others, 1995; Rigor and Colony, 1997). Surface waves are also part of a feedback mechanism where reduced ice extent leads to increased fetch and, therefore, increased wave height, which in turn results in the break-up of more of the polar sea ice (Thomson and Rogers, 2014).

Several types of ice are found in polar environments, which affect wave propagation in different ways. Ice floe fields exhibit hydrodynamic interaction with the incoming wave field as well as interaction between adjacent floes (Meylan and Squire, 1996). Continuous ice sheets interact with waves by imposing specific pressure and velocity boundary conditions at the water surface, which are related to flexural effects in the ice and lead to wave damping and modifications in the dispersion relation (Liu and MolloChristensen, 1988; Squire and others, 1995; Squire, 2007; Sutherland and Rabault, 2016). Grease ice and pancake ice accumulate and form a viscous layer that strongly attenuates surface waves (Weber, 1987; Keller, 1998). These thinner ice types are in their greatest abundance at the ice margins where wave interaction is strongest and ice-wave interactions the least understood. For these reasons, we focus our work here on these ice types along the marginal seas.

To clarify, grease ice is composed of frazil ice crystals, typically disks of size 1-4 mm in diameter and 1-100 $\mu \mathrm{m}$ in thickness (Newyear and Martin, 1997). Grease ice formation has been reported in cold areas where supercooled water is kept from freezing by surface turbulence (Newyear and Martin, 1997; De la Rosa and Maus, 2012). Grease ice accumulates and forms slicks of typical thickness $10-20 \mathrm{~cm}$
(Smedsrud and Skogseth, 2006; Smedsrud, 2011), that effectively damp high-frequency waves, therefore, appearing visually similar to an oil slick (Newyear and Martin, 1997).

The interactions between ice covers and incoming waves have been studied in previous works (Weber, 1987; Squire and others, 1995; Squire, 2007; Sutherland and Rabault, 2016), and several models have been presented. Massloading models only take into account the additional inertial effects originating from the presence of the ice and predict a reduction in the wavelength, but do not account for any damping (Peters, 1950). These models are insufficient when describing wave propagation in grease ice (Newyear and Martin, 1997). Similarly, the thin elastic plate model (Greenhill, 1886; Liu and Mollo-Christensen, 1988) describes the influence of flexural rigidity of the ice on wave propagation, which causes an increase of the wavelength but no damping. Such a model was developed to emulate a continuous unbroken ice sheet, but found to be unsuitable for grease ice (Newyear and Martin, 1997). Neither of these models predict wave attenuation, which needs to be described by separate mechanisms. Such mechanisms can be of two kinds: wave scattering, which is especially important in the case of the marginal ice zone (Kohout and Meylan, 2008); and the introduction of an effective viscosity in either the water or the ice layer (Weber, 1987; Keller, 1998; Carolis and Desiderio, 2002). The introduction of viscosity was initially proposed by Weber (1987) and refined by several authors later on (Keller, 1998; Carolis and Desiderio, 2002; Wang and Shen, 2010b). This last class of models is able to successfully reproduce laboratory observations of wave damping by grease ice, considering the effective viscosity as a fitting parameter. Therefore, it is this last class of models that we will examine in this paper.

More explicitly, the one-layer model of Weber (1987) assumes that the viscosity in the upper ice layer is high enough for the momentum equation to be reduced to 'a 
balance between pressure and friction (creeping motion)'. To explain, the effect of the creeping motion is to 'effectively halt the horizontal motion in the lower fluid at the interface' (Weber, 1987), and the viscous solution of Lamb (1932) is then recovered in the infinitely deep lower layer. An effective eddy viscosity much higher than the molecular viscosity of water is required in the water layer for the model to be consistent with observations and laboratory experiments. This viscous model of wave damping was later extended by Keller (1998), who describes the top layer by means of the incompressible Navier-Stokes equation, while the finite depth lower layer is described by the incompressible Euler equation. The solution in both layers is then obtained by linearizing about the state of rest. This two-layer model is much heavier mathematically than the one-layer model and features two parameters (ice thickness and effective viscosity of the ice layer) if taking the water depth and densities in each layer as given, instead of only one for the one-layer model (effective viscosity).

More recently, Carolis and Desiderio (2002) included an effective viscosity also in the lower layer, therefore introducing a third parameter in the model. The most recent refinement of this class of models was presented by Wang and Shen (2010b), who use a viscoelastic equation to describe the ice layer and model the water below as an inviscid layer. The formulation of Wang and Shen (2010b) went further to include both the flexural and the damping effects of the ice cover in the same model. However, these last models also suffer from a much increased mathematical complexity, which makes them difficult to use in comparisons with field data (Mosig and others, 2015).

Complementary to the numerous modeling efforts mentioned above, laboratory experiments have been performed in parallel to the development of theoretical models. The trends and theoretical curves obtained from the models are presented in the literature and compared with experimental results as a way to assess the quality of each model. However, quantitative metrics are currently not available in the literature to firmly establish the accuracy of any such model, making it difficult to objectively compare the quality of existing published predictions. Initial measurements in grease ice found good qualitative agreement (Newyear and Martin, 1997) with the one-layer model of Weber (1987). The two-layer model was later found to produce better qualitative agreement with experimental data (Newyear and Martin, 1999), but at the cost of more complex mathematics and the need for an additional parameter (ice thickness), as previously explained. The data from both experiments were used also by Carolis and Desiderio (2002) to validate their extended two-layer model with laboratory data. More recently, the effect of a mixed grease-pancake ice field was presented by Wang and Shen (2010a), which led the authors to the introduction of a viscoelastic description of the ice layer. The viscoelastic ice model was finally tested in laboratory experiments involving a variety of mixtures of grease and pancake ice (Zhao and Shen, 2015), and the authors concluded that it did a reasonable qualitative job fitting the observations, given that both equivalent viscosity and shear modulus are fitted using least-squares fits separately for each ice type.

While the development of more sophisticated models is impressive there are some drawbacks to this approach as the corresponding models grow in mathematical complexity and a greater number of fitting parameters is necessary. These advances are making it possible to obtain better qualitative agreement with laboratory and field results by enriching the underlying mathematics, but in the end the parameters used in all models are determined from empirical fit to experimental data (Wang and Shen, 2010a; Zhao and Shen, 2015). Such an approach only visually improves the quality of the model fits by adding more fitting coefficients, and pinpoints the need for the use of relevant quantitative metrics when comparing models. In addition, the wave modes for the two-layer models are obtained numerically from solving nonlinear dispersion relations, which are satisfied by infinitely many roots. Choosing the right wave mode requires some decision criterion, which proves challenging in the intermediate frequency range in particular when a viscoelastic model is used (Wang and Shen, 2010b; Mosig and others, 2015).

Moreover, articles about wave attenuation by grease ice report a wide range of empirical best-fit effective viscosity values. In their initial article, Newyear and Martin (1997) report frequency-dependent effective viscosity in the water layer using the model of Weber (1987) in the range $v_{\mathrm{w}}=$ $1.35-2.22 \times 10^{-2} \mathrm{~m}^{2} \mathrm{~s}^{-1}$, depending also on the grease ice thickness. In their second article, Newyear and Martin (1999) need to use an effective viscosity for the grease ice layer in the range $v_{i}=2.5-3.0 \times 10^{-2} \mathrm{~m}^{2} \mathrm{~s}^{-1}$ to describe experimental data with the two-layer model of Keller (1998). According to Carolis and Desiderio (2002), such variability arises from the fact that effective viscosities model both ice properties and other phenomena at the origin of wave damping, including the turbulence-driven dissipation. Carolis and Desiderio (2002) present a summary of eddy viscosities obtained from field measurements, that range from $\nu_{\mathrm{f}}=1.6-2.0 \times 10^{-2} \mathrm{~m}^{2} \mathrm{~s}^{-1}$ in the Weddell sea, where a high level of turbulence is present, down to $v_{\mathrm{f}}=0.24 \times$ $10^{-2} \mathrm{~m}^{2} \mathrm{~s}^{-1}$ in the central Arctic Ocean where much less turbulent conditions are observed. This high variability in eddy viscosity, and therefore in wave damping and effective viscosity, has also been observed for the same geographic region over just a few days (Doble and others, 2015). Attempts to provide theoretical justifications for the value of the effective viscosity needed to reproduce the damping observed in experiments have been presented (De Carolis and others, 2005), but cannot yet replace empirical fit to experimental data.

The diversity of models and parameter values discussed in the previous paragraphs makes it challenging to get a clear understanding of the situation. Two main questions arise in this context. First is the issue of how representative of the field conditions the different studies presented are. There is variability in the damping for waves in ice, and probably several different damping regimes are possible in the ocean. Therefore, characterizing the conditions in which each damping regime is observed is important. This will only be possible through the collection of much larger volumes of field data than have been discussed up to now (Mosig and others, 2015). While the number of articles that have been published could suggest that much data are available, articles discussing field data often rely on relatively small datasets and the data available on the whole are very scarce compared with the spatial extent of polar regions and diversity of regimes observed. Therefore, a first possible approach for clarifying the situation would be to collect 
much more field data. This in turn puts a sharp constraint on the acceptable cost of each measurement. We propose a possible solution for this issue, namely the use of instruments based on off-the-shelf sensors and open-source electronics as a way to reduce costs and increase measurement flexibility.

The second issue is to decide, confronted by the diversity of models presented in the literature, how the appropriate level of model complexity should be selected. Articles published so far have mostly introduced new and ever more complex models, fitted their parameters to a dataset and presented curves offering a visual impression of the quality of the fit. However, going further into determining which model to use should rely on some quantitative metrics that describe the statistical quality of each model. We address this issue by using several of the simplest model-fitting quality estimators, namely the coefficient of determination (Rao, 1973), usually noted $R^{2}$, and the mean absolute error (MAE) and RMSE. $R^{2}$ is a number between 0 and 1 that indicates the part of the variance in the data that are explained by the model, so that an $R^{2}$ value of 1 indicates that the model fully describes the variance in the data, while an $R^{2}$ value of 0 indicates that the model fails in explaining anything of the data presented. The MAE and RMSE are other well-established metrics that can be used to quantify the quality of a model (Willmott, 1982; Chai and Draxler, 2014), and that directly measure the discrepancy between model predictions and experimental data.

The organization of the paper is as follows. We first describe the architecture of the instruments used for the measurements. Next we present the data obtained and the methodology used to compare it with the one-layer model used by Weber (1987) and Newyear and Martin (1997). Finally, we present our results and discuss the agreement between our data and the one-layer model, as well as with the laboratory experiment of Newyear and Martin (1997), regarding the value of the effective viscosity observed.

\section{INSTRUMENTATION}

The harsh Arctic environment sets demanding requirements for scientific observations. While several commercial solutions are available (companies selling instruments operating in the Arctic include, e.g. Sea Bird Scientific Co., Campbell Scientific Co., Aanderaa Data Instruments A.S.), these usually come at high cost and have reduced flexibility. By contrast, off-the shelf sensors and open-source electronics are now sufficiently evolved, well documented and easy to use that they become a credible alternative to traditional solutions. The specificity of open-source software and electronics is that their source code, internal designs and interfaces are made available through a license that provides the right to study, modify and distribute the product (Joshua, 2014). This has many valuable implications for the scientific community. In particular, sharing all the details of the design of an instrument can make it easier to reproduce experiments, by drastically reducing the cost and time necessary to build an exact copy of the instrument initially used. In addition, this makes it easier to build upon a common platform, therefore encouraging modularity and reuse of previous designs rather than fragmented in-house development, which very likely is redundant between research groups and private suppliers, leading to unnecessary costs.
In the present case, we can choose a sensor with the characteristics required for performing measurements in the Arctic (including thermal calibration between -40 and $+85^{\circ} \mathrm{C}$ ), while reducing sharply the cost of each logger. Our approach consists in selecting the most cost-effective off-the-shelf sensor able to perform the measurements we need, and to build the whole logger around it using only open-source electronics and software. In our experience, common electronics components which rely on integrated circuits, flash memory and other semiconductors work in a wide range of temperature and, unlike batteries and sensors, do not present major problems in cold environments. Other groups in oceanography have started using open-source solutions, and the use of open-source electronics has been increasingly reported in the literature in the last few years (Baker, 2014; Gandra and others, 2015; Cauzzi and others, 2016). However, the solutions presented so far lack the generality, robustness and flexibility that will make them easily adaptable to a wide variety of projects. There is, therefore, a need for more groups to start sharing their designs together with detailed documentation, which would help to gather momentum around the use of opensource solutions in ocean research and create an opensource ecosystem similar to what has been achieved with, for example, scientific libraries around the Python language. In summary, transition toward at least partially open-source instrumentation can lead to a drastic reduction in price and development time for all groups working with field measurements, while at the same time making reproducibility of experiments by peers easier.

The data obtained in the present study were collected using an off-the-shelf sensor and an open-source logging system, which was created with generality and ease of modification in mind. The logger is based on a microcontroller rather than a traditional computer. This allows reduced price, power consumption and complexity. In addition an open-source GPS chip and SD card reader are integrated in the logger for absolute time reference, position information and data storage capability. Any type of sensor can then be added to build an instrument in a modular fashion.

The sensor model used to measure waves is the rugged, thermally calibrated VN100 Inertial Motion Unit (IMU) manufactured by Vectornav Co. This IMU was already assessed and used to produce valuable data in the field (Sutherland and Rabault, 2016). The VN100 is configured to output magnetic vector, linear accelerations, angular rates, temperature, pressure and checksum in ASCII format at $10 \mathrm{~Hz}$.

The IMUs and additional electronics were enclosed in hard plastic cases, to which a float was attached for ensuring that the whole system follows the waves well. More details about the technical solutions used for building the wave sensors are given in the Appendix.

\section{OBSERVATIONS}

Three instruments were deployed at sea near Longearbyen, Svalbard on 17 March 2016. A small boat was used to access the edge of a grease ice slick near shore to deploy the instruments. The water in the measurement area is shallow, down to $\sim 80 \mathrm{~cm}$ at the point closest to the shore. Wind waves generated locally came approximately perpendicular to the shore, and approximately parallel to a seawall limiting the extend of the grease ice slick to the East. The influence of the seawall on the waves recorded by the 


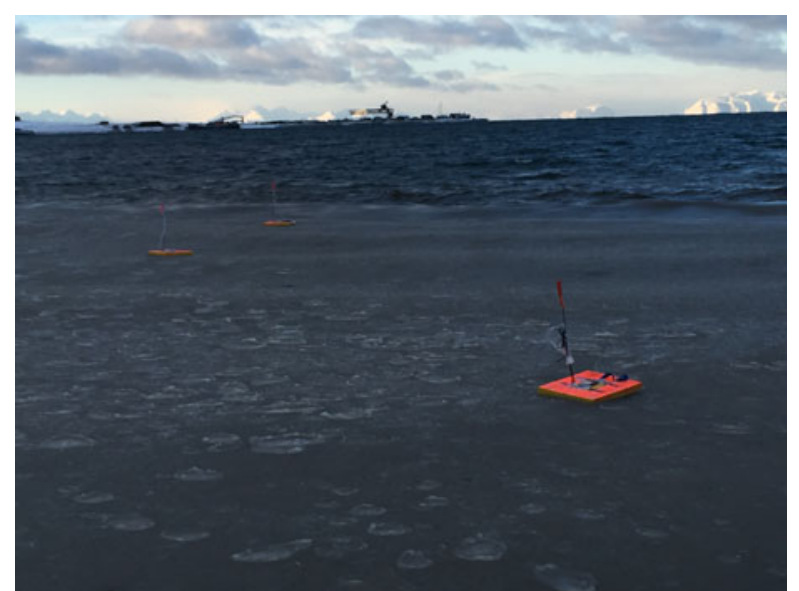

Fig. 1. Sensors F1, F2, F3 (by order of increasing distance from the camera) deployed near shore in a grease ice slick near Longyearbyen, Svalbard.

sensors was negligible. To allow easy recovery, the instruments were tied together with a rope, so that the maximum distance allowed between two instruments tied together was $\sim 15 \mathrm{~m}$. The total $30 \mathrm{~m}$ rope length used was long enough to not influence the dynamics of the floats and the maximum distance effectively measured between two instruments tied together is $\sim 8 \mathrm{~m}$, which indicates that there was no tension on the rope when measurements are performed. A picture of the ice slick and floats is presented in Figure 1. As visible in Figure 1, the instrument F1 entered the grease ice slick, while instruments F2 and F3 remained close to each other, at the limit of the slick during most of the measurement. The position of the instruments, obtained from the recorded GPS data, is presented in Figure 2. In all the following, the instruments are referred to as F1, F2 and F3. After 1

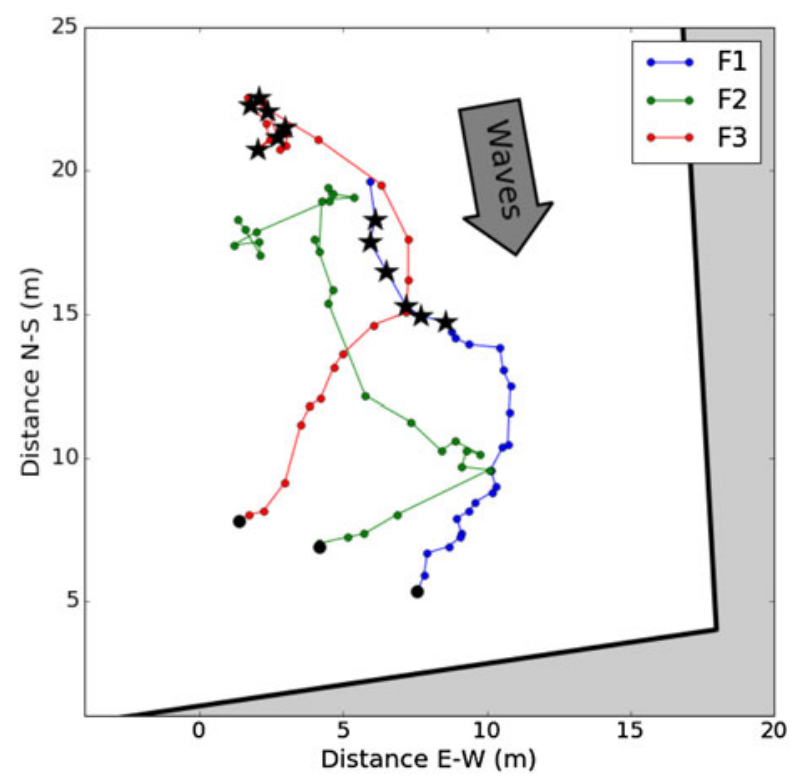

Fig. 2. Tracks of the wave sensors F1, F2 and F3 as recorded by their on-board GPS during the whole time of the measurements (UTC 14.10-16.30). Shore is indicated by the gray area. The positions of instruments F1 and F3 at the times of the beginning of each data sample used for computing wave attenuation by grease ice are marked with black stars. The final position of each instrument is indicated by a black dot.

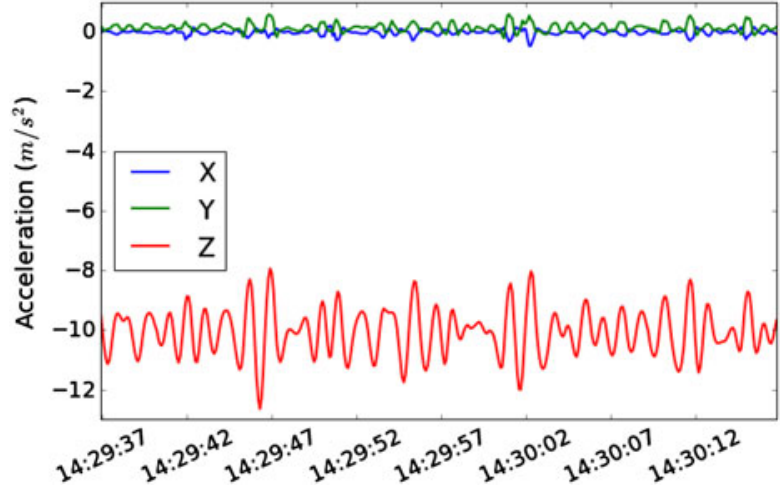

Fig. 3. Sample of the raw data for linear acceleration logged by the sensor F3. The time indicated is UTC on 17 March 2016. Data labeled $Z$ correspond to the IMU axes pointing upwards, while data labeled $X$ and $Y$ correspond to the two IMU axes in the horizontal plane.

h of drifting, F1 got grounded on shore and the wave data obtained by F1 are therefore not reliable after 15.05 UTC.

A sample of the raw data corresponding to the linear accelerations recorded by the instrument F3 is presented in Figure 3 . The factory-rated accuracy of the linear acceleration expected from the VN100 is $5 \times 10^{-3} \mathrm{~g}$, with $\mathrm{g}=$ $9.81 \mathrm{~m} \mathrm{~s}^{-2}$ the acceleration of gravity, which is $\sim 2.5$ orders of magnitude better than the typical vertical accelerations corresponding to the waves encountered during our measurements in grease ice. Therefore, as visible in Figure 3, the IMU is able to smoothly resolve the wave signal. The measured accelerations are primarily in the vertical direction (Z-axes of the IMU), while only small residual horizontal accelerations are observed ( $X$ - and $Y$-axes of the IMU). This was also observed visually during the deployment of the sensors, with the instruments appearing to be effectively stuck in the horizontal direction relatively to incoming waves due to the viscous grease ice layer.

The $10 \mathrm{~Hz}$ sampling frequency is well above the highest wave frequency observed, which is $\sim 1.2 \mathrm{~Hz}$. Therefore, the signal is effectively over sampled with respect to the Nyquist criterion in terms of water wave frequencies observed. While we record wave signal at $10 \mathrm{~Hz}$, the IMU works internally at $800 \mathrm{~Hz}$, so that the signal obtained is the result of a low-pass filtering done by the IMU processor and therefore eliminates otherwise possible aliasing of wind induced high-frequency accelerations.

\section{METHODOLOGY}

When computing the wave elevation power spectral density (PSD), the true vertical direction aligned with gravity is first determined by averaging the acceleration over the whole time series for each sensor. The maximum deviation compared with the Z-direction of the IMUs fixed inside the instrument cases is $<5^{\circ}$. The vertical wave acceleration is then obtained for each instrument by projecting the linear acceleration recorded by the IMU on the true vertical acceleration. We tested the effect of the angular deviation by adding to the vertical data an artificial random deviation of similar magnitude as the one experimentally measured, and processing the altered signal in the same way as presented in this section. We could not observe any significant influence on the results, as reported in the next section. The PSD of the 
wave vertical acceleration is computed with the Welch method (Earle, 1996) on 20-min intervals, using three time windows with $50 \%$ overlap, and low-pass filtered using a sliding average filter of width eight points. Error bars for the Welch spectra are computed using the Chi-squared $\left(\chi^{2}\right)$ error estimate. The PSD for the wave elevation is finally computed from the PSD of the wave vertical acceleration using the formula from Tucker and Pitt (2001):

$$
\operatorname{PSD}[\eta]=\omega^{-4} \operatorname{PSD}\left[\eta_{\mathrm{tt}}\right]
$$

where $\omega=2 \pi f$ is the angular frequency, $f$ the frequency, $\eta$ the wave elevation and $\eta_{t t}$ the vertical acceleration recorded by the IMU.

Since the PSD is quadratic in the wave amplitude, we can write the attenuation coefficient for the wave amplitude between $\mathrm{F}_{i}$ and $\mathrm{F}_{j}$ at each frequency, $a_{i / j}$, as:

$$
a_{i / j}(f)=\sqrt{\frac{\operatorname{PSD}_{i}(f)}{\operatorname{PSD}_{j}(f)}},
$$

where $\mathrm{PSD}_{i}$ is the PSD corresponding to the floating instrument $F_{i}$. The direction of the incoming waves, which is approximately perpendicular to the coast line, is at an angle $10^{\circ}$ west of north. The projection of the distance $d_{i / j}$ between instruments $F_{i}$ and $F_{j}$ on the wave propagation direction is therefore computed as:

$$
d_{i / j}=\boldsymbol{D}_{10} \cdot \boldsymbol{r}_{i / j}
$$

with $\boldsymbol{D}_{10}$ the wave propagation direction, and $\boldsymbol{r}_{i / j}$ the position vector from $\mathrm{F}_{i}$ to $\mathrm{F}_{j}$, computed from the GPS data recorded by the instruments.

The wave damping coefficient $\alpha(f)$ is defined for an incoming monochromatic wave of frequency $f$ as:

$$
A_{f}(x)=A_{f}(0) e^{-\alpha(f) x}
$$

with $A_{f}(x)$ the monochromatic wave amplitude at a distance $x$ along the wave direction of propagation, and $x=0$ is an arbitrary reference position. The damping coefficient $\alpha$ is therefore computed from the attenuation coefficient and the projected distance between $\mathrm{F}_{i}$ and $\mathrm{F}_{j}$ as:

$$
\alpha(f)=-\frac{\log \left(a_{i / j}(f)\right)}{d_{i / j}} .
$$

As emphasized in the introduction, several theoretical models describing wave attenuation by an ice cover have been proposed in the literature. While ice conditions can be controlled in laboratory experiments and grease ice thickness and effective viscosity can be measured and used as parameters in a two-layer model, it was not possible to measure those parameters during our field measurements. We could arbitrarily pick up a value for the grease ice layer thickness corresponding to a typical grease ice slick, and use this with one of the more sophisticated two-layer models. However, there would then be no advantage over the simpler one-layer models as the point of two-layer models is to account explicitly for the properties of the ice layer that are otherwise hidden in the value of the effective viscosity. This conflicts with the use of default values for the ice layer properties. Indeed, if default values for the ice layer properties are used based on a coarse assessment of the field conditions, all the fitting of the two-layer models is done based on the value of the effective viscosity of the ice or water layer, similarly to a one-layer model. Therefore, we compare our results with the one-layer model of Weber (1987), which has proven to yield satisfactory agreement with long waves at sea (Weber, 1987) and short waves in the laboratory (Newyear and Martin, 1997). The wave solution described by the one-layer model is the deep water limit of the more general viscous wave damping solution described by Lamb (1932). The generic equation describing the damping rate for any water depth, $\alpha_{\mathrm{g}}$ is (Lamb, 1932):

$$
\alpha_{\mathrm{g}}(f)=\frac{v k}{2 c_{\mathrm{g}} \delta}
$$

with $v$ the viscosity that in the original derivation is the viscosity of the fluid, but that can be replaced by an effective eddy viscosity in the water layer $v_{\mathrm{w}}$ to model field data, $k$ the wavenumber, $c_{\mathrm{g}}=\partial \omega / \partial k$ the group velocity, and $\delta=$ $\sqrt{2 v / \omega}$ the thickness of the Stokes layer.

The one-layer model is obtained by substituting the deep water linear dispersion relation, $\omega^{2}=g k$ with $g=9.81 \mathrm{~m}^{2}$ $\mathrm{s}^{-1}$ the acceleration of gravity, in Eqn (6). The expression for the damping rate using an effective viscosity in the water layer is then (Weber, 1987):

$$
\alpha(f)=\frac{\nu_{\mathrm{w}}^{1 / 2} \omega^{7 / 2}}{\sqrt{2} g^{2}} .
$$

Dissipation at the sea bottom is neglected in this model, which is justified as long as viscous dissipation due to the grease ice layer is the dominant dissipation mechanism. This hypothesis can be checked a posteriori. A formula describing the damping effect of the bottom and side wall boundary layers in a wave tank is provided by Sutherland and others (2017) as:

$$
\alpha_{\mathrm{bs}}=\nu \gamma k \frac{[(1 / \sinh (2 k H))+(1 / k B)]}{C_{\mathrm{g}}},
$$

where $\gamma=1 / \delta, H$ is the water depth, $B$ is the width of the wave tank and $v$ is the viscosity of water. In our case with sea water at $0^{\circ}$ Celsius $v=1.83 \times 10^{-6} \mathrm{~m}^{2} \mathrm{~s}^{-1}$. In the open sea, if one assumes that the seabed is smooth enough that the boundary layer there is similar to what is expected in a wave tank, only the first term in Eqn (8) will contribute since no side walls are present. The effect of the boundary layers on wave damping on both the experiment of Newyear and Martin (1997) and our field measurements will be analyzed in the Discussion section.

\section{RESULTS}

Figure 4 shows samples of the observed PSDs. The waves recorded are locally generated wind waves, with a peak frequency $\sim 0.8 \mathrm{~Hz}$ during the whole record. The damping effect of the grease ice slick is clearly visible. While instruments F2 and F3 remain close to each other at the limit of the grease ice slick and present little damping in most of the spectra, the instrument F1 that moved into the grease ice shows clear reduction in the wave PSD, especially at high frequencies as is expected from theory (Weber, 1987; Keller, 1998). However, F1 drifts to shallow areas as time 

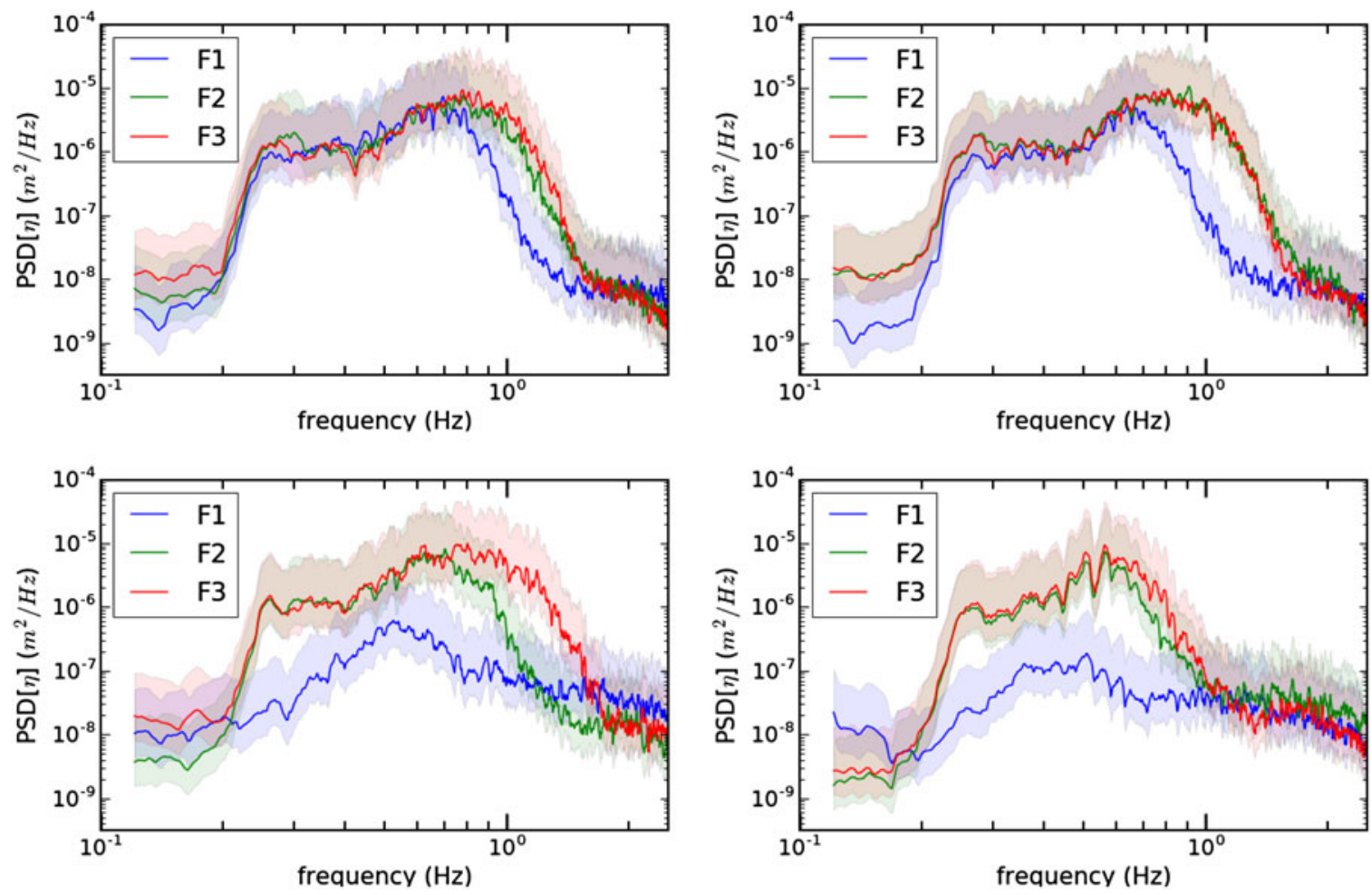

Fig. 4. Evolution with time of the PSD for wave elevation recorded by the sensors F1, F2 and F3 computed using the Welch method on 20-min intervals, using three time windows and $50 \%$ overlap followed by a sliding average filter. From left to right, top to bottom, the UTC time obtained from the internal GPS for the beginning of the time series used is $14.15,14.25,15.05,15.25$.

goes on and was grounded when recovered. This explains the excessive attenuation observed for times 15.05 and 15.25 UTC, and the corresponding PSD obtained from F1 should not be trusted.

During the time interval between 14.15 and 15.00 UTC, F1 is about $7 \mathrm{~m}$ further in the grease ice slick compared with F3, so that reliable wave damping coefficients can be computed. The location of the instruments during this time window is presented in Figure 2. During this time interval we can compute the damping based on the PSD for F1 and F3.

The value of the effective viscosity in the water layer yielding best agreement between Eqn (7) and laboratory experiments of Newyear and Martin (1997) is of the order of $\left(1.35 \pm 0.08 \times 10^{-2}\right) \mathrm{m}^{2} \mathrm{~s}^{-1}$ at $1.173 \mathrm{~Hz}$ for a $11.3 \mathrm{~cm}$ grease ice layer thickness (Table 1 of Newyear and Martin (1997)). Using the same model Eqn (7), we compute the best-fit

Table 1. Comparison between the results obtained by Newyear and Martin (1997) in the laboratory and our field measurements in Svalbard

\begin{tabular}{lll}
\hline & $\begin{array}{l}\text { Experiments by Newyear } \\
\text { and Martin (1997) }\end{array}$ & $\begin{array}{l}\text { Field measurements } \\
\text { in Svalbard }\end{array}$ \\
\hline $\begin{array}{l}\text { Frequency domain } \\
(\mathrm{Hz})\end{array}$ & 1.173 & $0.35-1.1$ \\
$\begin{array}{c}\text { Best-fit effective } \\
\text { viscosity }\left(\mathrm{m}^{2} \mathrm{~s}^{-1}\right)\end{array}$ & $1.35 \pm 0.08 \times 10^{-2}$ & $0.95 \pm 0.05 \times 10^{-2}$ \\
$R^{2}$ & $\mathrm{~N} / \mathrm{A}$ & \\
$\mathrm{MAE}\left(\mathrm{m}^{-1}\right)$ & $\mathrm{N} / \mathrm{A}$ & 0.89 \\
RMSE $\left(\mathrm{m}^{-1}\right)$ & $\mathrm{N} / \mathrm{A}$ & 0.037 \\
& & 0.044 \\
\hline
\end{tabular}

$R^{2}$, MAE and RMSE are computed based on the attenuation coefficient. We include only the measurement by Newyear and Martin (1997) at the frequency closest to the frequency range observed in the field. effective viscosity using nonlinear least-squares fit on the attenuation rate obtained through Eqn (5) for the collection of signal sample start times 14.20, 14.25, 14.30, 14.35, 14.40 and 14.45 UTC. We find a value $v_{\mathrm{w}}=(0.95 \pm 0.05 \times$ $\left.10^{-2}\right) \mathrm{m}^{2} \mathrm{~s}^{-1}$ using a 5- $\sigma$ confidence interval for the viscosity spread, which corresponds to a relative difference to the value found in previous cold laboratory experiments of $(30 \pm 10 \%)$. Propagating the angular deviation of the true vertical signal leads to relative variations in $v_{\mathrm{w}}$ of $<1 \%$. We also compute the coefficient of determination of our model on the field data and obtain a value of $R^{2}=0.89$. Finally, we compute the value of the MAE and RMSE relative to the prediction of the attenuation parameter, which are 0.037 and $0.044 \mathrm{~m}^{-1}$, respectively. Results are summarized in Table 1.

\section{DISCUSSION}

Obtaining a closed form wave damping formula from Eqn (6) requires the use of the deep water dispersion relation, as the more general intermediate water depth dispersion relation is not invertible in terms of exact known functions. However, the deep water approximation, which is already not strictly enforced in the laboratory study by Newyear and Martin (1997), is not strictly justified at the lower frequencies we report here. Therefore, we also compare the damping obtained in the field with Eqn (6), using the same effective viscosity in the water layer $v_{\mathrm{w}}=0.95 \times 10^{-2} \mathrm{~m}^{2} \mathrm{~s}^{-1}$ and the intermediate water depth dispersion relation:

$$
\omega^{2}=g k \tanh (k H)
$$

where $H=0.8 \mathrm{~m}$ is the water depth. Newyear and Martin $(1997,1999)$ showed that the effect of the grease ice layer 

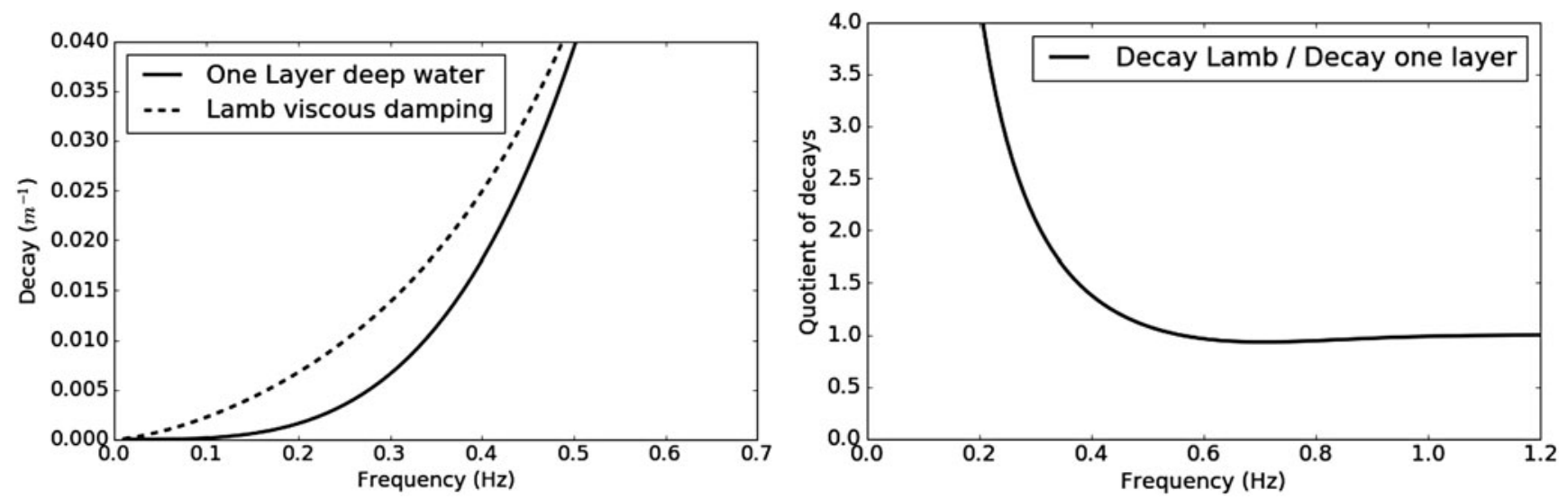

Fig. 5. Illustration of the effect of the choice of the deep or intermediate water depth dispersion relation on the wave attenuation obtained from Eqn (6). One layer label indicates that the formula from Weber (1987) which relies on the deep water dispersion relation is used, while Lamb label indicates that the intermediate water depth dispersion relation is used. Left: comparison of the attenuation curves for low frequencies. Right: quotient of both attenuation curves.

on the real part of the dispersion relation, i.e. changes in the wavelength introduced by the grease ice layer, can be neglected up to $\sim 1.2 \mathrm{~Hz}$, which is over the higher frequency limit for which we are able to compute damping. Therefore, we can use Eqn (9) to compute wavelength and group velocity even in the presence of a grease ice layer.

In the limit of low-frequency waves, the difference between the deep and intermediate water depth dispersion relations becomes important. As shown in Figure 5(a), both dispersion relations lead together with Eqn (6) to a zero attenuation in the low-frequency limit. However, the predicted quotient of the two attenuation curves goes to infinity in the low-frequency limit as shown in Figure 5(b), indicating that the velocity at which convergence to zero happens is very different between the two curves. As can be seen in Figure $5(b)$, the damping predicted by both dispersion relations is similar down to a frequency of $\sim 0.4 \mathrm{~Hz}$, which is slightly above the minimum frequency measured in the field, before diverging sharply. However, the damping in the frequency domain below $0.4 \mathrm{~Hz}$ for which both dispersion relations yield significantly different predictions is much smaller than for higher frequencies, and therefore the absolute difference between both predictions has a small impact on the least-squares fit used for obtaining the effective viscosity.

Results of the damping values obtained from the field measurements, compared with the one-layer model using the fitted effective viscosity value, are presented in Figure 6 . The thin lines indicate damping obtained from Eqn (5) for the six signal samples equally spaced in time, with start times between 14.20 and 14.45 UTC, that were used to fit the effective viscosity. The gray area indicates the $3 \sigma$ confidence interval based on all the damping curves. The thick black line indicates the one-layer model prediction from Eqn (7) obtained with the effective viscosity value previously reported, and the dashed line the intermediate water depth prediction from Eqn (6) using the same viscosity. Observations of wave attenuation in the field are therefore consistent with both the one-layer model of Weber (1987) and previous experiments in the laboratory.

We find good agreement, both qualitatively and quantitatively, between the one-layer model and the field data as shown by the width of the confidence intervals obtained, the value of the $R^{2}$ coefficient and the visual inspection of
Figure 6. By contrast, we obtain a bigger discrepancy between the effective viscosity computed from our measurements and obtained by Newyear and Martin (1997). Several explanations can be attempted to explain the corresponding $(30 \pm 10 \%)$ discrepancy. Firstly, the effective viscosity reported by Newyear and Martin (1997) increases slightly with frequency, which they attribute to a possible nonNewtonian behavior of the ice layer, so that reproducing their experiment for a lower frequency range corresponding to the field data obtained may yield better agreement. Secondly, as was emphasized in the introduction, the literature does report intrinsic variability in the effective viscosity due to, among other things, the thickness of the grease ice layer or the level of turbulence in the water. Those variables are difficult to measure in the field, and would be challenging to obtain from remote sensing and include in a wave model, therefore not testable at this time. As a consequence it is satisfactory to find that the one-layer model, which models all

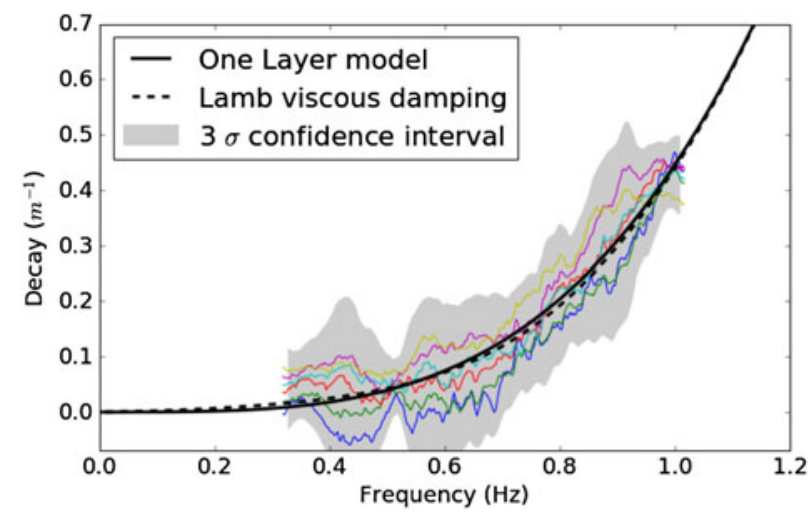

Fig. 6. Comparison of the observed damping rate from experimental data with the one-layer model. The thin curves show the frequencydependent damping rate obtained by comparing the PSD for wave elevation obtained from sensors F1 and F3 for six equally spaced data samples with start times between 14.20 and 14.45 UTC. The gray area is the corresponding $3 \sigma$ confidence interval. The thick line is the prediction of the one-layer model, with an effective viscosity in the water layer $v_{\mathrm{w}}=0.95 \times 10^{-2} \mathrm{~m}^{2} \mathrm{~s}^{-1}$. The dashed line is the prediction of the more general Lamb viscous damping solution, computed for the same effective viscosity as the onelayer model using the intermediate water depth dispersion relation. 
those effects in one single parameter (the effective viscosity), manages to produce similar results between field measurement and laboratory experiments, at least for the present small-scale study.

Another cause of discrepancy between our field measurements and laboratory results could be friction on the wave tank side walls. As explained previously, it is well known that the effects of the boundary layers on a wave tank side and bottom walls can affect wave damping, and must be accounted for to accurately compare laboratory results with theories (Sutherland and others, 2017). We can therefore use Eqn (8), together with the intermediate water depth dispersion relation (9), to estimate the effect of the seabed boundary layer on the measurements we performed in Svalbard, and of both the wave tank bottom and side walls in the case of the experiment by Newyear and Martin (1997). We use the viscosity of water at $0^{\circ} \mathrm{C}$ when computing the effect of the boundary layers. Results are presented in Figure 7. The effect of the seabed (or bottom wall) on wave decay has a peak at an intermediate frequency that depends on the water depth, and diminishes for higher frequency waves as wave motion gets concentrated near the surface. By contrast, the effect of the side walls increases with higher frequencies.

A comparison between the results presented in Figures 6 and 7 shows that the effect of the seabed on wave damping is about three orders of magnitude smaller than the effect of the grease ice at $0.6 \mathrm{~Hz}$. Therefore, even considering that the seabed roughness may increase the damping effect on the field data, the effect of the seabed boundary layer on wave decay can be neglected. In the case of the experiments by Newyear and Martin (1997), Figure 7 shows that while the effect of the side walls increases at higher frequencies, it is there also about two orders of magnitude smaller than the decay coefficient reported in their Table 1. However, the presence of ice at the water surface could

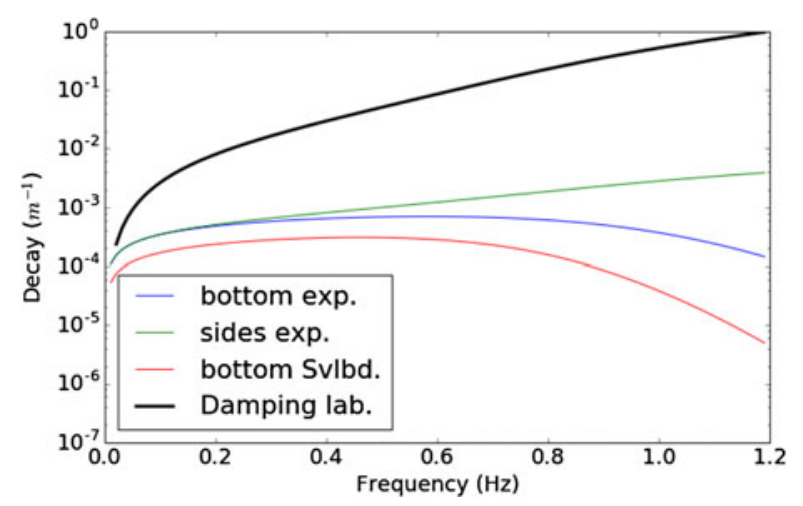

Fig. 7. Wave damping arising from the bottom and side walls in the experiment by Newyear and Martin (1997) (bottom exp. and sides exp. curves, respectively), along with the effect of the seabed boundary layer on the measurements we report in Svalbard (curve bottom Svlbd.). Decays are computed using Eqn (8) together with the intermediate depth dispersion relation Eqn (9) and the viscosity of water at $0^{\circ} \mathrm{C}$. According to the information provided by Newyear and Martin (1997), we use a water depth $H=0.5 \mathrm{~m}$ and a wave tank width $B=1 \mathrm{~m}$ in the laboratory data case. The water depth used in the field data case is $H=0.8 \mathrm{~m}$. The damping predicted by the one-layer model using the effective viscosity found by Newyear and Martin (1997) together with the dispersion relation for waves in intermediate water depth is presented for comparison (curve Damping laboratory). add another source of friction on the wave tank walls, which is not accounted for here. Unfortunately, we would need to perform a direct measurement of the friction coefficient of the grease ice on the material used for building the walls of the wave tank used by Newyear and Martin (1997) to assess the magnitude of this effect.

\section{CONCLUSIONS}

In the present paper, wave sensors are deployed in a grease ice slick near the shore in Svalbard and successfully measure wave spectra and wave attenuation by the grease ice. We present a comparison with the one-layer model of Weber (1987) in the frequency range $0.4-1 \mathrm{~Hz}$. The value of the effective viscosity in the water layer giving the best fit to the experimental data is $v_{\mathrm{w}}=\left(0.95 \pm 0.05 \times 10^{-2}\right)$ $\mathrm{m}^{2} \mathrm{~s}^{-1}$, which is $(30 \pm 10 \%)$ less than the value found at slightly higher frequency in previous cold laboratory experiments. We discuss the possible origin of this discrepancy, and cannot find any significant effect of the boundary layers developing on the wave tank walls and bottom. Therefore, we expect that the discrepancy arises from variability in the grease ice properties and the slightly lower frequency observed in the field compared with the laboratory.

In addition to the value of the effective viscosity, we obtain a coefficient of determination $R^{2}=0.89$, and values for the MAE and RMSE relative to the prediction of the attenuation parameter of 0.037 and $0.044 \mathrm{~m}^{-1}$, respectively. This proves that, in realistic conditions corresponding to field data where only partial information is available, so that sophisticated two-layer models could not be applied, the simpler one-layer model can still provide valuable information about wave damping. Using quantitative metrics such as the coefficient of determination, the MAE and RMSE could be a method to objectively compare different models for wave attenuation by grease ice.

The need for more field measurements for wave attenuation by ice has been underlined previously in the literature (Mosig and others, 2015). In this context, the instruments used for performing this work may also be of interest for other groups. Indeed, we built instruments that are versatile and based on off-the-shelf sensors and open-source electronics, which could make experiments both cheaper and easier to replicate by peers. Code and hardware details are shared as opensource material on the Github of the corresponding author (more details in the Appendix). It is hoped that such an opensource policy can become the norm for field measurements.

\section{ACKNOWLEDGMENTS}

The help of Aleksey Marchenko during the field work is gratefully acknowledged. The work was funded through the project 'Experiments on Waves in oil and ice' (Petromaks 2, Grant 233901). The authors want to thank two anonymous reviewers whose feedback greatly improved the initial manuscript. More information about the loggers can be found in the Appendix or on the Github of the first author (https:// github.com/jerabaul29/LoggerWavesInlce).

\section{REFERENCES}

Baker E (2014) Open source data logger for low-cost environmental monitoring. Biodivers. Data J., 2, e1059 (doi: 10.3897/BDJ.2. e1059) 
Carolis GD and Desiderio D (2002) Dispersion and attenuation of gravity waves in ice: a two-layer viscous fluid model with experimental data validation. Phys. Lett. A, 305(6), 399-412 (doi: http:// dx.doi.org/10.1016/S0375-9601(02)01503-7)

Cauzzi C and 5 others (2016) An open-source earthquake early warning display. Seismol. Res. Lett., 87 (doi: 10.1785/ 0220150284)

Chai T and Draxler RR (2014) Root mean square error (RMSE) or mean absolute error (MAE)? Arguments against avoiding RMSE in the literature. Geosci. Model Dev., 7(3), 1247-1250 (doi: 10.5194/gmd-7-1247-2014)

Chang $\mathrm{M}$ and Bonnet $\mathrm{P}$ (2010) Monitoring in a high-arctic environment: some lessons from MANA. IEEE Pervasive Comput., 9(4), 16-23 (doi: http://doi.ieeecomputersociety.org/10.1109/MPRV. 2010.53)

Christensen K and Broström G (2008) Waves in sea ice. Technical Report, Norwegian Meteorological Institute, Oslo

De Carolis G, Olla P and Pignagnoli L (2005) Effective viscosity of grease ice in linearized gravity waves. J. Fluid Mech., 535, 369-381 (doi: 10.1017/S002211200500474X)

De la Rosa S and Maus S (2012) Laboratory study of frazil ice accumulation under wave conditions. Cryosphere, 6(1), 173-191 (doi: 10.5194/tc-6-173-2012)

Doble MJ, De Carolis G, Meylan MH, Bidlot JR and Wadhams P (2015) Relating wave attenuation to pancake ice thickness, using field measurements and model results. Geophys. Res. Lett., 42(11), 4473-4481 (doi: 10.1002/2015GL063628), 2015GL063628

Earle MD (1996) Nondirectional and directional wave data analysis procedures. DBC Tech. Doc 96-01, National Data Buoy Centre, National Oceanic and Atmospheric Administration, U.S. Department of Commerce, Washington, DC.

Gandra M, Seabra R and Lima FP (2015) A low-cost, versatile data logging system for ecological applications. Limnol. Oceanogr.: Methods, 13(3), 115-126 (doi: 10.1002/lom3.10012), e10012

Greenhill AG (1886) Wave motion in hydrodynamics. Am. J. Math., 9(1), 62-96

Joshua MP (2014) Open-Source Laboratory, Elsevier, Amsterdam

Keller JB (1998) Gravity waves on ice-covered water. J. Geophys. Res., 103, 7663-7669 (doi: 10.1029/97JC02966)

Kohout AL and Meylan MH (2008) An elastic plate model for wave attenuation and ice floe breaking in the marginal ice zone. J. Geophys. Res.: Oceans 113(C9), n/a-n/a (doi: 10.1029/ 2007JC004434), c09016

Lamb H (1932) Hydrodynamics. Cambridge Mathematical Library, Cambridge University Press, Cambridge

Liu AK and Mollo-Christensen E (1988) Wave propagation in a solid ice pack. J. Phys. Oceanogr., 18(11), 1702-1712 (doi: 10.1175/ 1520-0485)

Meylan MH and Squire VA (1996) Response of a circular ice floe to ocean waves. J. Geophys. Res.: Oceans, 101(C4), 8869-8884 (doi: 10.1029/95JC03706)

Mosig JEM, Montiel F and Squire VA (2015) Comparison of viscoelastic-type models for ocean wave attenuation in icecovered seas. J. Geophys. Res.: Oceans, 120(9), 6072-6090 (doi: 10.1002/2015JC010881)

Newyear K and Martin S (1997) A comparison of theory and laboratory measurements of wave propagation and attenuation in grease ice. J. Geophys. Res.: Oceans, 102(C11), 25091-25099 (doi: 10.1029/97JC02091)

Newyear K and Martin S (1999) Comparison of laboratory data with a viscous two-layer model of wave propagation in grease ice. J. Geophys. Res.: Oceans, 104(C4), 7837-7840 (doi: 10.1029/ 1999JC900002)

Peters AS (1950) The effect of a floating mat on water waves. Commun. Pure Appl. Math., 3(4), 319-354 (doi: 10.1002/ сра.3160030402)

Pfirman S, Eicken H, Bauch D and Weeks W (1995) The potential transport of pollutants by arctic sea ice. Sci. Total Environ., 159
(23), 129-146 (doi: http://dx.doi.org/10.1016/0048-9697(95) 04174-Y)

Rao CR (1973) Linear statistical inference and its applications. Wiley, New York (doi: 10.1002/9780470316436)

Rigor I and Colony R (1997) Sea-ice production and transport of pollutants in the laptev sea, 19791993. Sci. Total Environ., 202(13), 89-110 (doi: http://dx.doi.org/10.1016/S0048-9697(97)00107-1), environmental Radioactivity in the Arctic

Smedsrud LH (2011) Grease-ice thickness parameterization. Ann. Glaciol., 52, 77-82 (doi: 10.3189/172756411795931840)

Smedsrud LH and Skogseth R (2006) Field measurements of arctic grease ice properties and processes. Cold Regions Sci. Technol., 44(3), 171-183 (doi: http://dx.doi.org/10.1016/j.coldregions.2005.11.002)

Squire V (2007) Of ocean waves and sea-ice revisited. Cold Regions Sci. Technol., 49(2), 110-133 (doi: http://dx.doi.org/10.1016/j. coldregions.2007.04.007)

Squire V, Dugan JP, Wadhams P, Rottier PJ and Ilu AK (1995) Of ocean waves and sea-ice. Annu. Rev. Fluid Mech., 27, 115168 (doi: http://10.1146/annurev..27.010195.000555)

Sutherland G and Rabault J (2016) Observations of wave dispersion and attenuation in landfast ice. J. Geophys. Res.: Oceans, 121(3), 1984-1997 (doi: 10.1002/2015JC011446)

Sutherland G, Halsne T, Rabault J and Jensen A (2017) The attenuation of monochromatic surface waves due to the presence of an inextensible cover. Wave Motion, 68, 88-96 (doi: http://dx. doi.org/10.1016/j.wavemoti.2016.09.004)

Thomson J and Rogers WE (2014) Swell and sea in the emerging arctic ocean. Geophys. Res. Lett., 41(9), 3136-3140 (doi: 10.1002/2014GL059983)

Tucker M and Pitt E (2001) Waves in ocean engineering. Elsevier ocean engineering book series, Elsevier, University of Michigan

Wadhams P and Doble MJ (2009) Sea ice thickness measurement using episodic infragravity waves from distant storms. Cold Regions Sci. Technol., 56(23), 98-101 (doi: http://dx.doi.org/10. 1016/j.coldregions.2008.12.002)

Wang R and Shen HH (2010a) Experimental study on surface wave propagating through a greasepancake ice mixture. Cold Regions Sci. Technol., 61(23), 90-96 (doi: http://dx.doi.org/10.1016/j.coldregions.2010.01.011)

Wang R and Shen HH (2010b) Gravity waves propagating into an ice-covered ocean: a viscoelastic model. J. Geophys. Res.: Oceans, 115(C6), n/a-n/a (doi: 10.1029/2009JC005591), c06024

Weber JE (1987) Wave attenuation and wave drift in the marginal ice zone. J. Phys. Oceanogr., 17(12), 2351-2361 (doi: 10.1175/ 1520-0485(1987)017<2351:WAAWDI > 2.0.CO;2)

Willmott CJ (1982) Some comments on the evaluation of model performance. Bull. Am. Meteorol. Soc., 63(11), 1309-1313 (doi: 10.1175/1520-0477(1982)063<1309:SCOTEO>2.0.CO;2)

Zhao X and Shen HH (2015) Wave propagation in frazil/pancake, pancake, and fragmented ice covers. Cold Regions Sci. Technol., 113, 71-80 (doi: http://dx.doi.org/10.1016/j.coldregions.2015.02.007)

\section{APPENDIX \\ TECHNICAL DETAILS ABOUT THE INSTRUMENTS USED}

The general architecture of the instruments is the following. An Arduino Mega microcontroller board is used together with a GPS chip, an active GPS antenna, and an SD card reader to build a modular instrument. The GPS chip communicates with the microcontroller through one of its four physical serial interfaces, while the SD card reader is wired on the SPI microcontroller bus. Sensors can then be added by simply plugging them into one of the three remaining 
Table 2. Summary of the power consumption of the electronics used, under active logging with $5 \mathrm{~V}$ power supply

\begin{tabular}{ll}
\hline Electronic component & Current consumption (mA at 5 V) \\
\hline Arduino Mega & 60 \\
SD card reader & 20 (dependent on SD card) \\
VN100 IMU & 70 \\
GPS and active antenna & 30 \\
Total & 180 \\
\hline
\end{tabular}

physical serial interfaces of the microcontroller, or by using the SPI or I2C bus. In addition, the GPS, SD card reader and sensors receive power through a MOSFET transistor that can be switched on and off from the microcontroller, which allows the whole instrument to be put in a lowpower consumption mode if requested.

Since the VN100 sensor used in our study relies on a RS232 $3 \mathrm{~V}$ level for serial communications while the microcontroller board uses TTL $5 \mathrm{~V}$ logic, a MAX232 logic converter chip is used for level conversion between the VN100 and the serial port of the microcontroller board used for logging. Data are logged directly in ASCII format at $10 \mathrm{~Hz}$. Binary format can be used to compress the data, but this was not necessary in our case and therefore ASCII was chosen for ease of programming and debugging. The current consumption of the whole system, when logging both the IMU at $10 \mathrm{~Hz}$ and GPS data at $1 \mathrm{~Hz}$, is $180 \mathrm{~mA}$ at $5 \mathrm{~V}$ (see Table 2). This is 20 times less than the overall power consumption that was needed for powering the MOXA computer-based instrument used in Sutherland and Rabault (2016).

Battery autonomy is the limiting factor for long-time logging in cold regions, which is made an even more critical issue than in temperate environments since the capacity of traditionally used lead acid batteries drops drastically in cold (Chang and Bonnet, 2010). We solve this issue by using industry grade rechargeable prismatic lithium iron (LiFe) batteries that feature low self-discharge and excellent performance in the cold. Two $40 \mathrm{Ah}, 3.2 \mathrm{~V}$ cells are assembled in series to provide a voltage of $6.4 \mathrm{~V}$, which is reduced to the $5 \mathrm{~V}$ needed by our electronics using a low dropout voltage regulator. In addition, a protection circuit module is inserted between the battery and the voltage regulator to prevent overloading or overdischarging. This solution was tested in the laboratory with the complete instrument including the VN100 IMU at a temperature of $-18^{\circ}$, and was able to work continuously for over 8 days. When only a few hours of logging are needed, we use more affordable lithium ion batteries.

To make the instrument resilient to failures caused by external events, such as short power interruptions due to shocks or other real work issues, a watchdog timer is used to reboot the microcontroller in case of a malfunctioning. In addition, the microcontroller logs the data to a new file every $15 \mathrm{~min}$, and the name of the previous file is stored in the nonvolatile EEPROM microcontroller memory to avoid erasing previous data even in the event of a reboot by the watchdog. This ensures that at most 15 min of data will be lost in case of a watchdog reset or power loss.

The microcontroller board, IMU, GPS chip and antenna, SD card reader and battery with protection circuit module are enclosed in a robust pelican case. The pelican cases are chosen, so that the whole system is buoyant, independently of any additional float.

Using microcontrollers presents several advantages over more powerful computer systems, such as the MOXA computer used in Sutherland and Rabault (2016). Price, power consumption, complexity, weight and size can be reduced, and therefore more sensors can be deployed for the same budget and can deliver longer autonomy (over 1 week of continuous measurements for an instrument weighting $<5 \mathrm{~kg}$ ) without operator intervention. Microcontrollers are not able to run sophisticated embedded processing, but they are able to perform logging and to interact with various sensors. More details about the code and the electrical components used are available on the corresponding author GitHub repository (https://github.com/jerabaul29/ LoggerWavesInlce). 